\title{
Traitement Expérimental Par Coagulation Floculation Des Effluents Brutes D'une Industrie Aéronautique À Casablanca (Maroc)
}

\author{
S. Namoussi, \\ Y. Errougui, \\ M. Chlaida, \\ A. Nahli, \\ C. Merbouh, \\ A. Naamane, \\ S. El Amrani, \\ N. Iounes, \\ Laboratoire d'Ecologie et d'Environnement (LEE), \\ Université Hassan II- Casablanca, Faculté des Sciences Ben M'sik, \\ Sidi Othmane, Casablanca, Maroc
}

Doi:10.19044/esj.2019.v15n36p402 URL:http://dx.doi.org/10.19044/esj.2019.v15n36p402

\section{Résumé}

Les effluents de l'industrie de traitement de surface présentent souvent une forte charge en produits chimiques très toxiques qui doivent être traités conformément à une réglementation de plus en plus stricte. Dans ce travail, nous avons effectué une caractérisation des effluents liquides d'une unité de traitement de surface au Maroc qui a révélé une forte pollution colloïdale (Turbidité=131 NTU), une charge en matière oxydable élevée (DCO = 960 mg/l) et en éléments métalliques (Fer, cuivre, zinc, nickel, aluminium, chrome et cyanure). L'objectif de cette étude expérimentale consiste à appliquer un traitement physico-chique par coagulation-floculation à ces eaux uses industrielles toxiques en vue de les conformer aux normes de rejet dans le réseau d'assainissement local. En effet, ce traitement, précédé d'un ajustement $\mathrm{du} \mathrm{pH}$, une oxydation des cyanures et d'une réduction du chrome hexavalent, a abouti à une réduction très satisfaisante de la toxicité de ces effluents avec des taux d'abattement de $94 \%$ pour la DCO et jusqu'à $99 \%$ pour la turbidité et charge métallique.

Mots clés : Traitement de surface, rejets, caractérisation, coagulation/floculation, métaux lourds 


\title{
Experimental Treatment By Coagulation Flocculation Of Raw Effluents From An Aerospace Industry In Casablanca (Morocco)
}

\author{
S. Namoussi, \\ Y. Errougui, \\ M. Chlaida, \\ A. Nahli, \\ C. Merbouh, \\ A. Naamane, \\ S. El Amrani, \\ N. Iounes, \\ Laboratoire d'Ecologie et d'Environnement (LEE), \\ Université Hassan II- Casablanca, Faculté des Sciences Ben M'sik, \\ Sidi Othmane, Casablanca, Maroc
}

\begin{abstract}
Effluents from the surface treatment industry often have a high load o f highly toxic chemicals that need to be treated in accordance with increasing ly stringent regulations. In this work, we performed a characterization of liqu id effluents from a surface treatment unit in Morocco that revealed high collo idal pollution (Turbidity=131 NTU), high stainless material (COD=960 mg/l ) and metal elements (Iron, copper, zinc, nickel, aluminum, chromium and cy anide). The objective of this experimental study is to apply a physico-chemical treatment by coagulation-flocculation to these toxic industrial wastewater in order to comply with the discharge standards in the local sewage system. This treatment, preceded by $\mathrm{pH}$ adjustment, cyanide oxidation and reduction of hexavalent chromium, resulted in a very satisfactory reduction in toxicity of these effluents with abatement rates of $94 \%$ for COD and up to $99 \%$ for turbidity and metal load.
\end{abstract}

Keywords: Surface Treatment, Discharges, Characterization, Coagulation/Fl occulation, Heavy Metals

\section{Introduction}

Les industries de traitement de surface font partie des secteurs d'activités industrielles les plus polluants car elles utilisent de nombreux 
produits chimiques en particulier à bas de métaux et de diverses substances organiques ou/et minérales caractérisés par leur toxicité pour l'homme et l'environnement (Yatribi et al., 2000 ; Druart et al.,2016 ;Zongo, 2009). En effet, les effluents générés par ces industries contiennent entre autres des résidus tels que les cyanures, acides, bases et des métaux lourds (Nikel, Zinc, Cadmium, Chrome ...)(Montvydienè et al., 2007; Crini et al., 2012; CARPP, 2002). Du fait de leur toxicité vis à vis de l'environnement, ces effluents industriels doivent être traités pour respecter les seuils imposés par la réglementation (SEMEMEE, 2010 ; MDMEMEE, 2014) avant leurs rejets au niveau du réseau d'assainissement ou dans le milieu naturel. Le traitement physico-chimique constitue souvent le procédé le plus appliqué pour éliminer ces polluants organiques et minéraux utilisés dans la plupart des industries de traitement de surface (Blais et al., 1999 ; Abid et al., 2010 ; Sancey et al., 2010).

$\mathrm{Au}$ Maroc, la problématique majeure de ce type d'effluents réside dans le fait qu'ils ne sont pas toujours traités par la majorité des industries, ce qui peut porter atteinte au réseau d'assainissement local et aux équipements des stations d'épuration urbaines auxquelles ses industries sont raccordées (Chlaida et al., 2011). Au même moment, il y a un risque de contamination des ressources hydriques aussi bien superficielles que souterraines (Bouderka et al., 2016) voir même marines (Ayah et al., 2015; Siba et al., 2018) et littorales (Izougarhane et al., 2016).

Ce travail s'intéresse au traitement des effluents liquides d'une filière de traitement de surface d'une industrie de fabrication de pièces aéronautiques située au niveau de la plateforme aéroportuaire Mohammed V à Casablanca. L'objectif est d'appliquer un traitement physico-chimique expérimental d'affinage par coagulation/floculation aux eaux usées issus des différents bains de traitement et rinçage. Auparavant, ces eaux usées, et avant leur rejet dans le réseau d'assainissement local, subissent un prétraitement de décyanuration/déchromatation pour éliminer les cyanures et transformer le chrome hexavalent $\left(\mathrm{Cr}^{6+}\right)$ en chrome trivalent $\left(\mathrm{Cr}^{3+}\right)$ en vue de se conformer aux valeurs limites stipulées dans les normes marocaines de rejets d'émission spécifiques aux industries de traitement de surface (SEMEMEE, 2010).

\section{Fonctionnement de la filière \\ Description du procédé de traitement de surface}

$\mathrm{Au}$ sein de cette unité industrielle, les pièces aéronautiques fabriquées subissent un traitement de surface au sein d'une série de cuves intégrées dans les chaînes de production de l'entreprise (Figure 1). Ces cuves fonctionnent soit comme des bains de traitement et ou des bains de rinçage des pièces fabriquées (Khyati et al., 2004). 


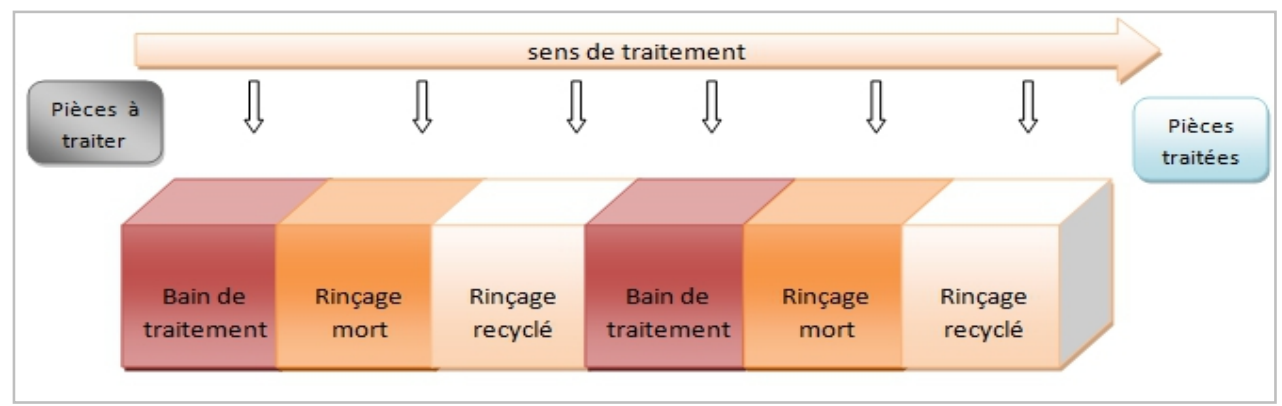

Figure : Composition générale de la chaîne de traitement de surface

Les produits utilisées au niveau des bains de traitement, qui se retrouvent généralement dans les eaux en sortie de chaîne de production, sont formés de plusieurs espèces chimiques : acides, bases, métaux (chrome, nickel, aluminium, Fer ...), cyanures, chrome hexavalent $\left(\mathrm{Cr}^{6}+\right)(\mathrm{Crini}$ et al., 2012). Quant aux bains de rinçage intercalés entre les différents bains de traitement, ils permettent de stopper et neutraliser les réactions chimiques et d'éviter également la contamination des bains avals (Laforest, 1999 ; Leveillard,2011).

Les différents polluants issus des différentes cuves de traitement varient suivant le type de procédé de production lié au substrat à traiter et à la composition des bains utilisés (Tireche, 2008). Les rejets les plus concentrés en polluants correspondent aux eaux des bains de traitement qui sont vidangés une fois les éléments chimiques se présentent hors tolérances de fonctionnement. Les rejets dilués sont essentiellement constitués des eaux issues des bains de rinçage (Tatangelo, 2006). L'analyse de la composition physico-chimique de ces effluents (Tableau $\mathrm{V}$ ) révèle une charge en matières oxydables (DCO), en matières en suspension (MES) et colloïdale (turbidité) importantes dépassant largement les valeurs limites de rejet stipulées dans les normes marocaines de rejets d'émission spécifiques aux industries de traitement de surface (SEMEMEE, 2010). Au même moment, le rapport $\mathrm{DCO} / \mathrm{DBO}_{5}$ reste très élevé (32) confirmant que les eaux usées générées son très loin d'être biodégradables d'autant plus que les teneurs des différents éléments métalliques, à l'exception du $\mathrm{Cu}$, s'avèrent élevées et non conformes aux normes précitées.

Sur le plan quantitatif, l'entreprise consomme annuellement environ $4041 \mathrm{~m}^{3}$ d'eau potable et génère parallèlement chaque année 2367,58 $\mathrm{m}^{3} \mathrm{~d}$ 'eaux usées issues des bains de traitement (soit $522,88 \mathrm{~m}^{3}$ dont $14,52 \mathrm{~m}^{3}$ sont cyanurés, $177,69 \mathrm{~m}^{3}$ sont chromiques et $330,67 \mathrm{~m}^{3}$ sont acido-basiques) et des bains de rinçage (soit $1853,7 \mathrm{~m}^{3}$ dont $775,10 \mathrm{~m}^{3}$ sont chromiques, 10,8 $\mathrm{m}^{3}$ sont cyanurés et $1067,80 \mathrm{~m}^{3}$ sont acido-basiques). 


\section{Traitement des rejets par décyanuration et déchromatation}

Le cyanure, encore couramment employé dans la composition des bains (CARPP, 2002), est un produit à caractère très toxique qu'on doit convertir en substance moins toxique (Pareau, 1995; Roques, 1998). La méthode la plus utilisée à l'échelle industrielle, est l'oxydation du cyanure $\left(\mathrm{CN}^{-}\right)$en cyanate $\left(\mathrm{CNO}^{-}\right)$par l'hypochlorite de sodium $(\mathrm{NaClO})$ à un $\mathrm{pH}$ supérieur à 9,5 selon la réaction globale (Tatangelo, 2006 ; Abid et al., 2010 ; Euvrard, 2017 ; Laforest ,1999)

\section{$\mathrm{NaCN}+\mathrm{NaClO} \rightarrow \mathrm{NaCNO}+\mathrm{NaCl}$}

La quantité d'hypochlorite de sodium utilisée, selon la concentration de cyanures à éliminer, a été déterminée suivant l'instruction contrôle de la société sous la référence IC-R5.05-098. Ainsi, pour l'oxydation d'1kg de cyanure $\left(\mathrm{CN}^{-}\right)$en cyanate $\left(\mathrm{CNO}^{-}\right)$, il convient d'utiliser 22 litres d'hypochlorite de sodium $(\mathrm{NaClO})$ commercial 47 à $50^{\circ}$ chlorométriques.

Le chrome, également employé dans la composition des bains de traitement (CARPP, 2002), est un produit à caractère toxique qui nécessite une déchromatation. La méthode la plus utilisée est la réduction du chrome hexavalent $\left(\mathrm{Cr}^{6+}\right)$ en chrome trivalent $\left(\mathrm{Cr}^{3+}\right)$ à l'aide du bisulfite de sodium $\left(\mathrm{NaHSO}_{3}\right)$ à pH acide entre 1,8 et 2,5 (Abid et al., 2010 ; Sancey et al., 2010). Cette réduction est marquée par un changement très net de la coloration de la solution des bains de traitement passant du jaune au vert. Le procédé a lieu selon la réaction chimique suivante (Tatangelo, 2006 ; Abid et al., 2010 ; Euvrard, 2017 ; Trunfio et al.,2010):

$$
4 \mathrm{H}_{2} \mathrm{CrO}_{4}+6 \mathrm{NaHSO}_{3}+3 \mathrm{H}_{2} \mathrm{SO}_{3} \rightarrow 2 \mathrm{Cr}_{2}\left(\mathrm{SO}_{4}\right)_{3}+3 \mathrm{Na}_{2} \mathrm{SO}_{4}+10 \mathrm{H}_{2} \mathrm{O}
$$

La quantité de bisulfite à appliquer, variable selon la concentration de l'acide chromique, a été fixée selon l'instruction contrôle de la société référencée ICR5.05-41. A titre indicatif, pour réduire $1 \mathrm{~kg}$ de chrome hexavalentCr ${ }^{6+}$ en chrome trivalent $\mathrm{Cr}^{3+}$ au niveau des eaux usées des bains de traitement, il faut faire appel à $3 \mathrm{~kg}$ de bisulfite de sodium.

Après traitement des eaux des bains à l'hypochlorite de sodium et au bisulfite de sodium, les cyanures et le chrome $\mathrm{Cr}^{6+}$ deviennent indétectables. Ce résultat, rapporté ailleurs (Abid et al., 2010), montre que ces effluents des bains de traitement sont conformes aux normes de rejet (SEMEMEE, 2010) pour ce qui est de ces polluants spécifiques mais ils restent particulièrement chargés en chrome trivalent $\left(\mathrm{Cr}^{3+}\right)$ et autres matières en suspension, oxydables et métaux divers.

\section{Matériel et méthodes}

\section{Procédé de traitement expérimental par coagulation/ floculation}

La coagulation-floculation est un procédé de traitement physicochimique utilisé pour éliminer les matières en suspension colloïdales (Semerjian et al, 2003). Lors de cette étude, les essais expérimentaux de 
coagulation-floculation sont appliqués directement sur des échantillons d'eaux usées brutes $(200 \mathrm{ml})$, issues des bains de traitement et de rinçage, ayant subi une décyanuration et et une déchromatation juste avant leur rejet dans le réseau d'assainissementlocal. Ces tests sont réalisés à l'aide d'un jar-test type VELP scientifica à quatre béchers, fonctionnant à une vitesse de rotation pouvant atteindre $200 \mathrm{tr} / \mathrm{min}$.

Les produits coagulant et floculant utilisés correspondent respectivement au chlorure ferrique $\mathrm{Fe} \mathrm{Cl}_{3}$ à $30 \%$ et à un polyacrylamide anionique. Ces produits sont appliqués à différentes doses, à des $\mathrm{pH}$ variables et à une vitesse de rotation/agitation du jar-test de $150 \mathrm{tr} / \mathrm{min}$. Le but est de déterminer les conditions optimales pour obtenir les plus gros flocs à décanter et l'eau la plus limpide et claire possible. Le résultat obtenu est évalué à travers la mesure de la turbidité de l'échantillon traité et le calcul du taux de son abattement.

\section{Mesures et analyses physico-chimiques}

Lors de cette étude, les échantillons ont été prélevés, durant la période allant du 01 Janvier au 30 Juin 2015, au niveau des quatre chaînes de production en pleine activité et au cours des divers cycles de production. Cet échantillonnage est effectué à un endroit où il y a suffisamment de turbulence/agitation pour assurer l'homogénéité de l'échantillon.

La caractérisation physico-chimique des eaux, réalisée après chaque test, porte sur la mesure des paramètres globaux $\left(\mathrm{T}^{\circ}, \mathrm{pH}\right.$, Turbidité, MES, $\left.\mathrm{DBO}_{5}, \mathrm{DCO}\right)$ et spécifiques notamment les cyanures et les métaux : $\mathrm{Fe}, \mathrm{Cu}, \mathrm{Zn}, \mathrm{Ni}, \mathrm{Al}$ et $\mathrm{Cr}$. Les méthodes de mesure et d'analyse des eaux usées, utilisées sont résumées dans le tableau I.

Tableau I : Matériels et méthodes de caractérisation des paramètres physico-chimiques

\begin{tabular}{|c|c|c|}
\hline Paramètre & $\begin{array}{c}\text { Matériels/méthod } \\
\text { e }\end{array}$ & Références \\
\hline Température $\left({ }^{\circ} \mathrm{C}\right)$ & Thermomètre & TH CA 004 \\
\hline $\mathrm{pH}($ Unités $\mathrm{pH})$ & $\mathrm{pH}$ mètre & $\mathrm{pH} \mathrm{3310} \mathrm{SET} \mathrm{2}$ \\
\hline La turbidité $(\mathrm{NTU})$ & Turbidimètre & EUTECH TN-100 \\
\hline Demande Chimique en & Thermoréacteur à & $\begin{array}{c}\text { Méthode DIN ISO } \\
15705\end{array}$ \\
\hline Oxygène "DCO" $(\mathrm{mg} / \mathrm{l})$ & kits type DCO10119 & Oxitopvelpsientifica( \\
\hline Demande Biochimique en & Méthode & AFNOR, 1999 - NFT \\
Oxygène en 5 jours "DBO5" $(\mathrm{mg} / \mathrm{l})$ & manométrique & $1899-2)$ \\
\hline Matières en Suspension & Filtration sur filtre & AFNOR, 1999-NF EN \\
"MES" (mg/l) & Wattman GFC \\
\hline Métaux $(\mathrm{Fe}, \mathrm{Cu}, \mathrm{Zn}, \mathrm{Ni}, \mathrm{Al}$, & Spectrophotométri & WTW Spectroflex \\
Cr) et Cyanures $(\mathrm{CN})$ & e aux kits standards & 6100 \\
\hline
\end{tabular}




\section{Résultats et discussions}

\section{Tests de traitement par coagulation et floculation}

Test de détermination de la dose optimale du coagulant

Lors de ce premier test de traitement destiné à déterminer la dose optimale du coagulant (chlorure ferrique à 30\%), la turbidité chute rapidement après addition du coagulant. Le meilleur résultat est obtenu à une dose de $6 \mathrm{ml}$ de chlorure ferrique, soit une turbidité de 10 NTU correspondant à un taux d'abattement de $92 \%$ (tableau II). Le pH final de la solution reste neutre et donc conforme aux valeurs limites de rejet (SEMEMEE, 2010 ; Lydec, 1998).

Tableau II : Résultats du test de coagulation/floculation pour détermination de la dose optimale du coagulant

\begin{tabular}{|c|c|c|c|c|c|}
\hline \multicolumn{2}{|c|}{ Echantillon $(200 \mathrm{ml})$} & $\begin{array}{c}1 \\
\text { (Échantillon brut) }\end{array}$ & 2 & 3 & 4 \\
\hline \multicolumn{2}{|c|}{ Dose du coagulant (ml) } & 0 & 4 & 6 & 9 \\
\hline \multicolumn{2}{|c|}{$\mathrm{pH}$ final } & 8,07 & 7,72 & 7 & 6 \\
\hline \multirow{2}{*}{ Turbidité } & NTU & 131 & 10,93 & 10 & 40,9 \\
\hline & \% d'abattement & - & $91 \%$ & $92 \%$ & $68 \%$ \\
\hline
\end{tabular}

Test de détermination du $\mathrm{pH}$ optimal

Lors de ce $2^{\circ}$ test de coagulation-floculation, la dose du chlorure ferrique appliquée à tous les échantillons est de $6 \mathrm{ml}$ et le $\mathrm{pH}$ a été modifié en ajoutant le lait de chaux à $10 \%$. Le meilleur taux d'abattement $(97 \%)$ de turbidité (2,96 NTU) est obtenu à un pH de 9,5 (tableau III). Mais ce pH optimal n'est pas conforme aux valeurs limites de rejet (SEMEMEE, 2010).

Tableau III : Résultats du test de coagulation/floculation pour la détermination du pH optimal

\begin{tabular}{|c|c|c|c|c|c|c|}
\hline \multicolumn{2}{|c|}{ Echantillon (200 ml) } & Echantillon brut & 1 & 2 & 3 & 4 \\
\hline \multicolumn{2}{|c|}{ Dose du coagulant $(\mathrm{ml})$} & 0 & 6 & 6 & 6 & 6 \\
\hline \multicolumn{2}{|r|}{$\mathrm{pH}$} & 8,07 & 8 & 9,5 & 5,76 & 11,2 \\
\hline \multirow{2}{*}{ Turbidité } & NTU & 131 & 45,6 & 2,96 & 9,56 & 90,63 \\
\hline & \% d'abattement & - & $65 \%$ & $97 \%$ & $92 \%$ & $30 \%$ \\
\hline
\end{tabular}

Test de détermination de la dose optimale du floculant

Au cours de ce test, les conditions optimales précédentes de dose du coagulant $(6 \mathrm{ml})$ et de $\mathrm{pH}(9,5)$ sont appliquées mais avec une variation des concentrations du floculant (polyacrylamide anionique). Le meilleur résultat de ce test, soit une turbidité de 1,68 NTU et un taux d'abattement de $99 \%$, est obtenu avec $6 \mathrm{ml}$ de coagulant et $4 \mathrm{ml}$ de floculant (tableau IV). Toutefois, un taux d'abattement élevé de la turbidité ( $98 \%$ ) est observé dès le départ du test sans coagulant $(0 \mathrm{ml})$ et avec $4 \mathrm{ml}$ de floculant. Ce résultat pourrait s'expliquer par le fait que le lait de chaux, ajouté pour optimiser le $\mathrm{pH}$ à 9,5, déclencherait un début de coagulation. 
Tableau IV : Résultats du test de coagulation/floculation pour la détermination de la dose optimale du floculant

\begin{tabular}{|c|c|c|c|c|c|c|c|}
\hline \multicolumn{2}{|c|}{ Echantillon $(200 \mathrm{ml})$} & Echantillon brut & 1 & 2 & 3 & 4 & 5 \\
\hline \multicolumn{2}{|c|}{ Dose de coagulant ml } & 0 & 0 & 6 & 6 & 6 & 6 \\
\hline \multicolumn{2}{|c|}{ Dose de Floculant ml } & 0 & 4 & 4 & 6 & 9 & 12 \\
\hline \multicolumn{2}{|c|}{ pH optimal } & 8,07 & 9,5 & 9,5 & 9,5 & 9,5 & 9,5 \\
\hline \multirow{2}{*}{ Turbidité } & NTU & 131 & 2,15 & 1,68 & 4,89 & 2,92 & 9,6 \\
\hline & $\%$ d'abattement & - & 98 & 99 & 96 & 97 & 92 \\
\hline
\end{tabular}

\section{Caractérisation physico-chimique des eaux usées}

Les résultats des analyses physico-chimiques des eaux traitées dans les conditions optimales du coagulant $(6 \mathrm{ml})$ et de floculant $(4 \mathrm{ml})$, montrent que les valeurs de tous les paramètres aussi bien globaux que spécifiques présentent des taux d'abattement importants allant jusqu'à $99 \%$ (Tableau V). Le Chrome trivalent $\left(\mathrm{C}^{3+}\right)$ issu du prétraitement de déchromatation est indétectable. Au même moment, toutes les teneurs enregistrées pour les autres paramètres globaux ou spécifiques restent largement inférieures aux valeurs limites de rejets spécifiques aux industries de surface (SEMEMEE, 2010). Ces résultats témoignent de l'efficacité du traitement par coagulation/floculation comme l'ont montré les travaux de Abid et al. (2010) avec une réduction de 95\% de la charge métallique ainsi que les travaux de Sancey et al. (2010) qui rapportent que ce traitement physico-chimique est capable de diminuer la charge polluante avec des taux d'abattement de $100 \%$ pour l'Argent et le Chrome et de $72 \%$ pour le Zinc. De même, les travaux de Charles et al. (2019) ont montré que le traitement par voie physico-chimique par coagulation-floculation est capable de diminuer la charge organique, les nitrites et le zinc avec des taux d'abattements respectifs de $80 \%, 90 \%$ et 95 $\%$. Nos résultats vont dans le même sens que ceux obtenus par Yatribi et al. (1999) avec un pourcentage d'abattement de $95 \%$ pour le chrome et $95 \%$ pour les matières en suspension. 
Tableau V: Résultats des analyses physico-chimiques des eaux usées traitées sous conditions optimales de coagulation/floculation en comparaison avec les valeurs limites de rejets

\begin{tabular}{|l|c|c|c|c|}
\hline & $\begin{array}{c}\text { Eau usée } \\
\text { brute }\end{array}$ & $\begin{array}{c}\text { Eau usée traitée par } \\
\text { coagulation/floculatio } \\
\text { n après } \\
\text { décyanuration- } \\
\text { déchromatation }\end{array}$ & $\begin{array}{c}\text { Abattemen } \\
\mathrm{t}(\%)\end{array}$ & $\begin{array}{c}\text { Valeurs } \\
\text { Limites de } \\
\text { Rejet } \\
\text { d'émission } \\
(\mathrm{mg} / \mathrm{l})[9]\end{array}$ \\
\hline $\mathrm{DBO}_{5}(\mathrm{mg} / \mathrm{l})$ & 30 & 26 & $13 \%$ & 100 \\
\hline $\mathrm{DCO}(\mathrm{mg} / \mathrm{l})$ & 960 & 52,9 & $94 \%$ & 500 \\
\hline $\mathrm{MES}(\mathrm{mg} / \mathrm{l})$ & 1330 & 32 & $97 \%$ & 50 \\
\hline $\mathrm{DCO} / \mathrm{DBO} 5$ & 32 & 2,03 & - & - \\
\hline Température $\left({ }^{\circ} \mathrm{c}\right)$ & 30 & 30 & - & 30 \\
\hline $\mathrm{pH}$ & 8,07 & 6,5 & - & $6-6,8$ \\
\hline Turbidité $(\mathrm{NTU})$ & 131 & 1,68 & $99 \%$ & 30 \\
\hline $\mathrm{Fe}(\mathrm{mg} / \mathrm{l})$ & 98 & 0,39 & $99 \%$ & 20 \\
\hline $\mathrm{Cu}(\mathrm{mg} / \mathrm{l})$ & 2 & 0,04 & $98 \%$ & 4 \\
\hline $\mathrm{Zn}(\mathrm{mg} / \mathrm{l})$ & 7 & 0,5 & $92 \%$ & 10 \\
\hline $\mathrm{Ni}(\mathrm{mg} / \mathrm{l})$ & 8 & 0,5 & $93 \%$ & 5 \\
\hline $\mathrm{Al}(\mathrm{mg} / \mathrm{l})$ & 11,4 & 4,7 & $58 \%$ & 10 \\
\hline $\mathrm{Chrome} \mathrm{Cr}{ }^{6+}(\mathrm{mg} / \mathrm{l})$ & 1,667 & Non détecté & $100 \%$ & 0,2 \\
\hline $\mathrm{CN}(\mathrm{mg} / \mathrm{l})$ & 2,45 & Non détecté & $100 \%$ & 0,2 \\
\hline
\end{tabular}

\section{Conclusion}

La caractérisation des effluents brutes de l'industrie aéronautique de la plateforme aéroportuaire Mohammed V à Casablanca, ayant fait l'objet de cette étude, révèle une forte charge en matières colloïdales et oxydable et présentant également une contamination en éléments métalliques (Fer, cuivre, zinc, nickel, aluminium ...). Bien que ces rejets subissent au préalable un prétraitement, par déchromatation et décynuration ciblant respectivement le chrome hexavalent et le cyanure, il s'est avéré qu'ils ne respectent pas les normes marocaines relatives aux rejets des industries de traitement de surface. A ce propos, l'application d'un procédé de traitement physico-chimique expérimental par coagulation/floculation, au chlorure ferrique à $30 \%$ et au polyacrylamide anionique, a donné des résultats très satisfaisants. En effet, en plus de l'élimination totale des cyanures et surtout du chrome $\mathrm{Cr}^{3+}$, ce traitement physico-chimique d'affinage a entrainé des abattements importants pour les autres paramètres atteignant jusqu'à $99 \%$ pour les MES, turbidité et la plupart des métaux. Au même moment, les eaux traitées répondent très largement aux valeurs limites de rejets spécifiques aux industries de surface au Maroc.

\section{References:}

1. Yatribi, A., Najmeddine, A. (2000). Impact écotoxicologique du traitement chimique des eaux usées de tanneries : analyse technico- 
économique. Revue Des Sciences de L'eau, Volume 13, N²(2000) p $107-118$

2. Montvydienè, D., Marčiulionienè, D. (2007).Assessment of toxic interaction of metals in binarymixtures using lepidiumsativum and spirodelapolyrrhiza.Polish Journal of Environmental Studies $\mathrm{N}^{\circ} 6(5) \mathrm{p}$ 777-783

3. Secrétariat d'état auprès du ministre de l'Energie des Mines de l'Eau et de l'Environnement chargé Eau "SEMEMEE". (2010). Arrêté fixant des valeurs limites spécifiques de rejet de la branche de galvanisation à chaud relevant de l'activité du traitement de surface. bulletin officiel $n^{\circ} 5836$ - 21 joumada i 1431(6-5-2010).

4. Ministère délégué auprès du ministre de l'énergie, des mines, de l'eau et de l'environnement, chargé de l'eau et de l'environnement département de l'eau "MDMEMEE". (2014).Préservation de la qualité des ressources en eau et lutte contre la pollution (valeurs limites de rejet à respecter par les déversements (normes de pollution).

5. Blais, J.F., Dufresne, S., Mercier, G. (1999). Etat du développement technologique en matière d'enlèvement des métaux des effluents industriels. Revue des sciences de l'eau. Volume 12, $\mathrm{N}^{\circ} 4(1999) \mathrm{p}$ 687-711

6. Aziza Abid, Abdeljalil Zouhri et Abdelali Ider. (2010). Dimensionnement d'une station automatique et continue de traitement physicochimique des rejets liquides de traitement de surface. Journal Afrique SCIENCE $\mathrm{N}^{\circ}$ 06(2) (2010) p93 - 107

7. Sancey, B., Trunfio, G., Charles, J., Minary, JF., Gavoille, S., Badot, PM., Crini, G. (2010). Heavy metal removal from industrial effluents by sorption on cross-linked starch: chemical study and impact on water toxicity.journal of environmental management $\mathrm{N}^{\circ}$ 92(3) (2011) p103-184-198.

8. Chlaida, M., Brand, C., Kraume, M., Moutaib, Z and Fouad, S. (2011 ).Wastewater in the peri-urban area of grand casablanca (morocco): status quo, treatment and potential reuse in urban agriculture in 3rd international symposium Re-Water Braunschweig , 21-22 November 2011, p265-281

9. Bouderka, N., Kacem SA., Lakhili, F., Lahrach, A., Benabdelhadi, M. (2016). L'impact De La Pollution Agricole Sur La Qualite Des Eaux Souterraines De La Nappe Du Gharb. European Scientific Journal N ${ }^{\circ}$ 11 Volume 12 p509-524

10. Ayah, M., Grybos, M., Tampo, L., Bawa, LM., Bril, H. (2015). Qualité Et Pollution Des Eaux D'un Hydrosystème Littoral Tropical: Cas Du Système Lagunaire De Lomé, Togo. European Scientific Journal $\mathrm{N}^{\circ} 15$ Volume 11 p 95-119 
11. Siba, A., Eljaafari, S., Mokhtari, F. (2018). Pollution Bactérienne Et Toxique Dans Les Eaux De Rejets Industriels Et Domestiques Du Littoral Atlantique (Casablanca Est- Maroc). European Scientific Journal N¹2 Volume 14 p283-296

12. Izougarhane,M., Mansouri, D., El Ibaoui1, H., Chakiri, S., Fadli, M. (2016). Physico-Chimie Et Teneurs Metalliques Des Eaux De L'estuaire De L'oued Sebou Durant Des Annees De Dragage Du Sable/ 2007, 2014, 2015, 2016. European Scientific Journal N³0 Volume 12 p127-147

13. Khyati, A., Messafi, A.(2004). Traitement des rejets liquides émanant des industries de traitements de surfaces et leur réutilisation dans les circuits des chaînes selon le principe « zéro rejet », journal desalination. p 87-99.

14. Crini, G., Gavoille, S. (2012).Bilan du programme metaldex 20082012. métaux lourds et rejets industriels: développement de nouveaux procédés de traitement des effluents industriels - évaluation de l'efficacité environnementale. UMR Chrono-environnement Agence de l'Eau Rhône Méditerranée \& Corse - 27 septembre 2012p 3-28

15. Laforest, V. (1999). Technologies propres : Méthodes de minimisation des rejets et de choix des procédés de valorisation des effluents : application aux ateliers de traitement de surface, Sciences de l'ingénieur .Ecole Nationale Supérieure des Mines de SaintEtienne; INSA de Lyon, 1999. Français, 280 p

16. Sihem Tireche, Abdelaziz Tairi. (2008). Evaluation of the impact of surface industry treatment on the quality of ouedisser. World Water Congress2008 Montpellier le 01-04 September 2008

17. Tatangelo, A. (2006). Optimisation de la précipitation des métaux lourds en mélange et valorisation des boues d'hydroxydes : application aux effluents de traitement de surfaces Ecole Nationale Supérieure des Mines de Saint-Etienne, 2006. Français. Ingénierie de l'environnement $293 \mathrm{p}$

18. Elise, E. (2017). Substances dangereuses dans les effluents de traitement de surface : identification et traitement par des procédés innovants Université de Franche-Comté, 2016. Français 369p

19. Pareau, D., Durand, G. (1995). Traitements D’effluents Industriels Contenant Des Traces De Cyanures Residuels Etude Record N910301

20. Roques, H. (1998). Fondements Théoriques Du Traitement Chimique Des Eaux, Technique Et Documentation Lavoisier Vol 2

21. Florine Leveillard. (2011). Methodologie de minimisation de la pollution appliquée au traitement de surface : Etude de l'entrainement 
comme vecteur de pollution Ecole Nationale Supérieure des Mines de Saint-Etienne, 2011. Français. $234 \mathrm{p}$

22. Centre d'activités régionales pour la production propre "CARPP", plan d'action pour la méditerranée. (2002). Alternatives de prévention de la pollution à la source dans le secteur du traitement de surfaces. 158p.www.cema-sa.org.

23. IC-R5.05-098

24. IC-R5.05-41

25. Trunfio, G., Crini, G. (2010). The déchromatation step in wastewater treatment plants: fundamental role and optimization.Ind. Eng. Chem. Res. Vol. 49, $\mathrm{N}^{\circ}$ 23(2010) p 12217-12223 IC-R5.05-41

26. Semerjian, L., Ayoub, GM. (2003). High-ph-magnesium coagulation-flocculation in wastewater treatment. Advances in Environmental Research $N^{\circ} 7$ (2003) p 389-403

27. Afnor. (1999). Recueil de normes françaises : la qualité de l'eau. tome $4 \mathrm{p} 128$.

28. Lyonnaise des eaux de Casablanca Lydec. (1998). Rejets liquides :Cahier des charges de LYDEC.

29. Charles, J., Crini , G., Sancey, B., Trunfio, G., Badot, PM., MorinCrini, N., Minary, JF., Gavoille, S., Lagarrigue, C., Torri, G., Vismara, E et Winterton, P. (2019). Suivi et optimisation d'une station de décontamination des eaux usées de la filière traitement de surface : abattement chimique et impact écotoxicologique. journal of water science Volume 24, $\mathrm{N}^{\circ}$ 4(2011) p. 329-341.

30. Yatribi, A., Nejmeddine, A. Bouguerne,A et Tifnouti, A. (1999) . Evaluation de la toxicité des eaux usées industrielles de tanneries impact du traitement chimique .journal déchets - sciences et techniques $\mathrm{N}^{\circ} 13$ (1999) p 36-40. 